\title{
An Effective Insulin Therapy in Combination With Sodium- Glucose Cotransporter 2 Inhibitors
}

\author{
Hidekatsu Yanai ${ }^{\mathrm{a}, \mathrm{b}}$, Hiroki Adachia ${ }^{\mathrm{a}}$, Mariko Hakoshima ${ }^{\mathrm{a}}$, \\ Hisayuki Katsuyama ${ }^{a}$
}

\section{To the Editor}

Recently, Suzuki et al. reported the comparison of combined tofogliflozin (sodium-glucose cotransporter 2 inhibitor, SGLT2i) and insulin glargine, tofogliflozin added to insulin, and insulin dose-increase therapy in uncontrolled type 2 diabetes. [1]. In the tofogliflozin + insulin group, approximately $90 \%$ of patients were treated by mixed insulin or bolus + basal insulin. In the glargine + tofogliflozin group, only basal insulin was used. After 24 weeks, HbAlc significantly decreased in the groups using tofogliflozin than in insulin alone group, suggesting that the addition of SGLT2i to insulin is superior to insulin alone with regard to glycemic control. Reduction of $\mathrm{HbA} 1 \mathrm{c}$ was non-significantly greater in the tofogliflozin + insulin group $(-1.04 \%)$ than in the glargine + tofogliflozin group $(-0.76 \%)$. However, reduction of daily insulin dose was significantly greater in the glargine + tofogliflozin group $(-12.67 \mathrm{U})$ than in the tofogliflozin + insulin group $(-2.56 \mathrm{U})$. Furthermore, body weight reduction was greater in the glargine + tofogliflozin group $(-3.4 \mathrm{~kg})$ than in the tofogliflozin + insulin group $(-2.9 \mathrm{~kg})$. In the report which studied effects of dapagliflozin on 24-h glycemic control in type 2 diabetic patients by using continuous glucose monitoring (CGM), the improvement of 24-h mean glucose was greater in the metformin group $(-36.2 \mathrm{mg} / \mathrm{dL})$ than in the insulin group (-11.9 $\mathrm{mg} / \mathrm{dL}$ ) [2]. The improvement in time in hyperglycemic range was also more pronounced in the metformin group (-15.3\%) than in the insulin group $(-8.9 \%)$. These recent studies let us think about an effective insulin therapy in combination with SGLT2i.

The meta-analyses showed that the addition of SGLT2i to insulin therapy significantly reduced fasting plasma glucose, $\mathrm{HbA1c}$, total daily insulin dose and body weight in both type 1 and type 2 diabetes $[3,4]$.

Manuscript submitted November 1, 2018, accepted November 13, 2018

a Department of Internal Medicine, National Center for Global Health and Medicine Kohnodai Hospital, Chiba, Japan

${ }^{\mathrm{b}}$ Corresponding Author: Hidekatsu Yanai, Department of Internal Medicine, National Center for Global Health and Medicine Kohnodai Hospital, 1-7-1 Kohnodai, Ichikawa, Chiba 272-8516, Japan.

Email: dyanai@hospk.ncgm.go.jp

doi: https://doi.org/10.14740/jocmr3676
SGLT2i caused a decrease in the renal threshold for glucose excretion $\left(\mathrm{RT}_{\mathrm{G}}\right)$, and an increase in urinary glucose excretion and a reduction in plasma glucose levels in both health individuals and diabetic patients $[5,6]$. Therefore, if plasma glucose levels were above $\mathrm{RT}_{\mathrm{G}}$, SGLT2 $\mathrm{i}$ was effective to decrease plasma glucose (Fig. 1a). However, if plasma glucose levels were below $\mathrm{RT}_{\mathrm{G}}$, SGLT2i did not work to decrease plasma glucose (ineffective range of SGLT2i) (Fig. 1b).

Studies which showed effects of the addition of SGLT2i to insulin therapy on insulin dose were shown in Table 1 [7-11]. Insulin reduced plasma glucose in a relatively dose-dependent manner in type 1 diabetic patients whose intrinsic insulin was depleted. However, SGLT2i reduced plasma glucose independently of insulin. Dose-dependent effects of SGLT2i on reduction of both bolus insulin and basal insulin were not observed in patients with type 1 diabetes $[7,8]$. In type 1 diabetic patients treated with insulin and SGLT2i, glycemic control may be determined by administered insulin dose and $\mathrm{RT}_{\mathrm{G}}$ reduced by SGLT2i. Interestingly, the reduction in basal insulin dose was small in type 1 diabetic group showing a large reduction in bolus insulin dose $[7,8]$. There was a tendency to show a negative correlation between changes in bolus insulin dose and basal insulin dose (Fig. 2), indicating that if bolus insulin dose was increased, basal insulin can be reduced and vice versa. When we treat type 1 diabetic patients, we should titrate bolus and basal insulin dose not to make plasma glucose below $\mathrm{RT}_{\mathrm{G}}$ (ineffective range of SGLT2i).

If patients who had the profile shown in Figure 3a took SGLT2i and showed a reduction in plasma glucose shown in Figure 3b, they can reduce both basal and bolus insulin doses like patients in the study by Matsumura et al [9]. If they show a reduction in plasma glucose as shown in Figure 3c, they can reduce only bolus insulin dose like patients in our previous study [10]. In type 2 diabetic patients whose intrinsic insulin was preserved, treated with insulin and SGLT2i, glycemic control may be determined by the following factors, 1) an improvement of insulin resistance due to body weight loss by SGLT2i; 2) an improvement of glucotoxicity by insulin and/or SGLT2i; 3) administered insulin dose; 4) $\mathrm{RT}_{\mathrm{G}}$ reduced by SGLT2i. If we can decrease plasma glucose to target plasma glucose levels by using basal insulin and SGLT2i and other oral anti-diabetic drugs (OADs) which can reduce postprandial glucose such as dipeptidyl-peptidase 4 inhibitors and alpha glucosidase inhibitor, we can stop using bolus insulin like Figure 3d. Furthermore, SGLT2i was reported to reduce insulin dose in patients 

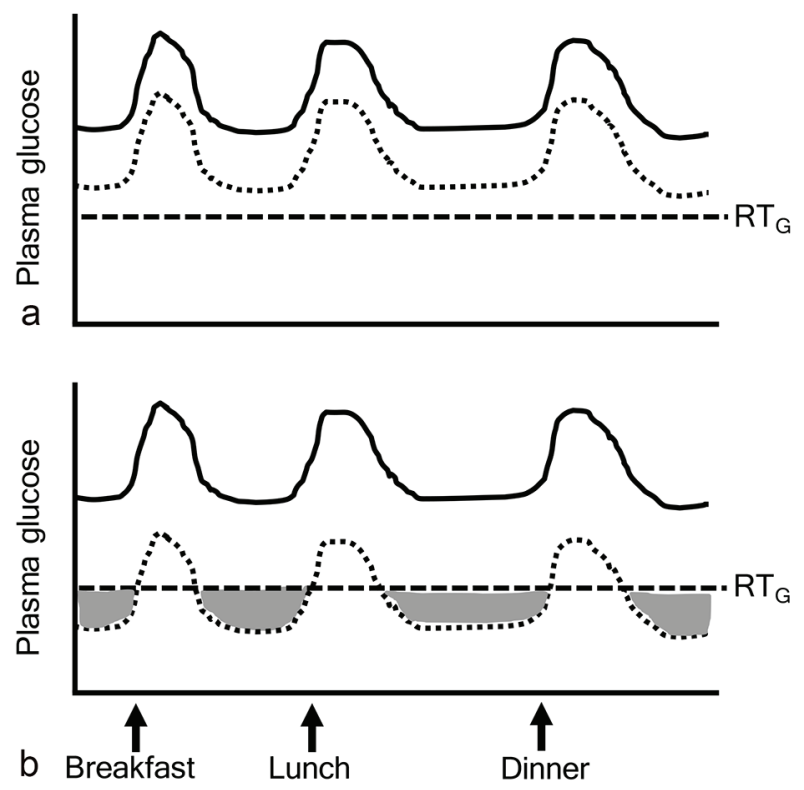

Figure 1. Ineffective range of SGLT2 inhibitors. The gray area indicates "ineffective range of SGLT2i" which shows that plasma glucose levels are below the renal threshold for glucose excretion $\left(R T_{G}\right)$. Solid and dotted lines indicate plasma glucose levels before and after the addition of SGLT2 inhibitors, respectively.

treated by using basal insulin and OADs [11]. As seen in the study by Suzuki et al [1], a larger reduction of insulin dose produced a greater decrease in body weight.

Basal insulin therapy was less likely to induce hypoglycemia as compared with bolus-basal insulin therapy, suggesting that basal insulin therapy was less likely to produce "ineffective range of SGLT2i" as compared with bolus-basal insulin therapy. The addition of SGLT2i to basal insulin therapy

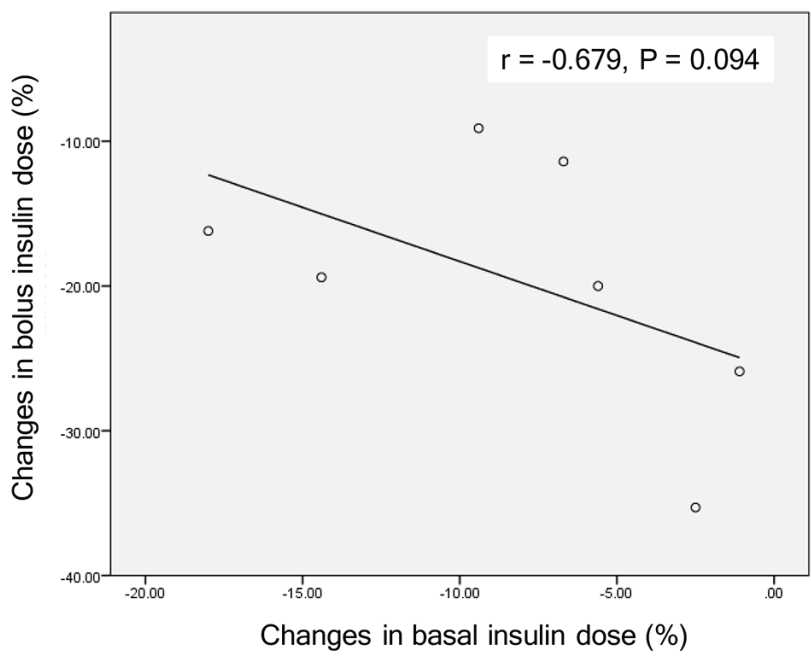

Figure 2. A correlation between changes in bolus insulin dose and changes in basal insulin dose by the addition of SGLT2 inhibitors in patients with type 1 diabetes. Correlation was analyzed by Spearman's correlation.

showed a greater reduction in $\mathrm{HbAlc}(-1.03 \%)$, insulin dose $(-1.58 \mathrm{U} /$ day $)$ and body weight $(-1.64 \mathrm{~kg})$ as compared with the addition of SGLT2i to bolus-basal insulin therapy $(-0.39 \%$, $-1.33 \mathrm{U} /$ day and $-1.18 \mathrm{~kg}$ ) [12], supporting our suggestion.

In conclusion, when we use insulin in addition to SGLT2i in both type 1 and type 2 diabetes, we should titrate insulin dose not to make "ineffective range of SGLT2i".

\section{Conflict of Interests}

The authors declare that they have no conflict of interest con-

Table 1. Insulin Therapy in Combination With SGLT2 Inhibitors in Type 1 and Type 2 Diabetes

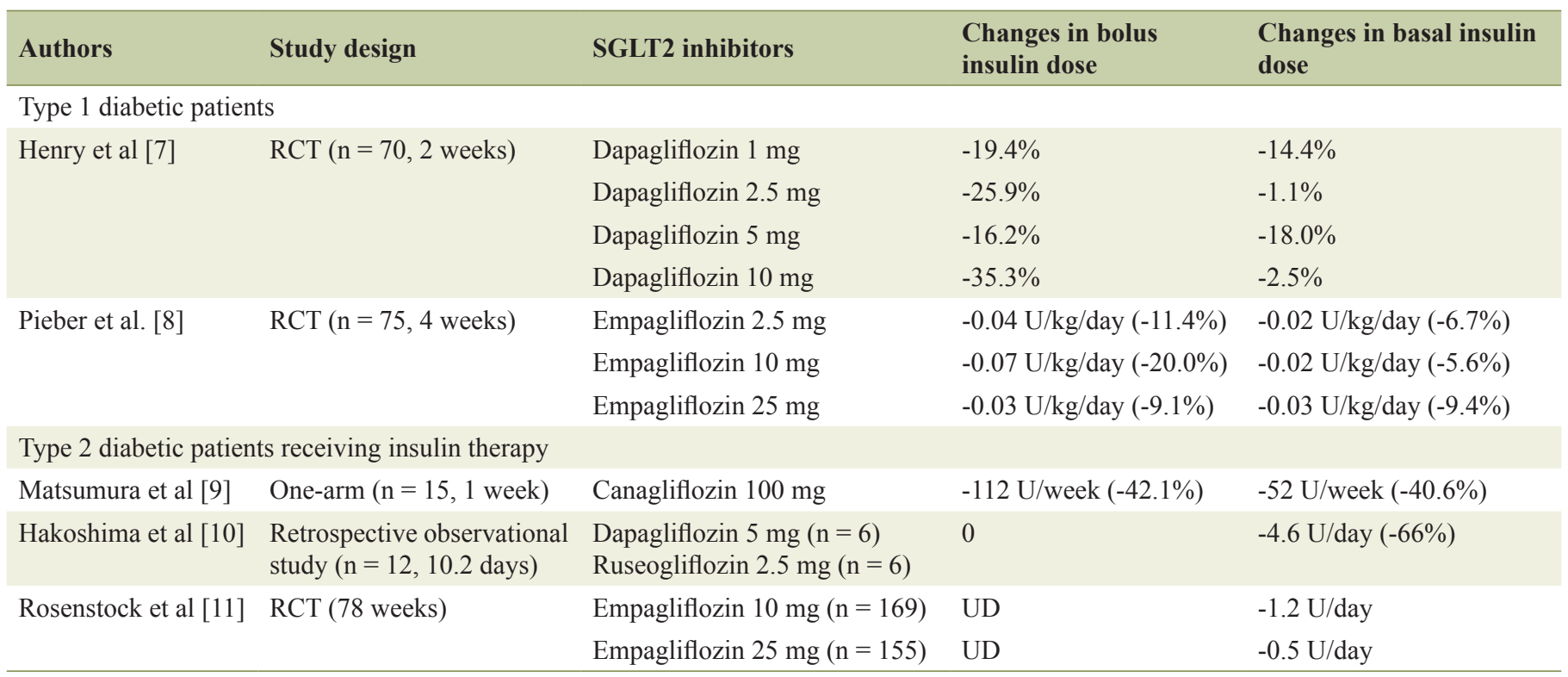

RCT, randomized, double-blind, placebo-controlled study; UD, undetermined. 


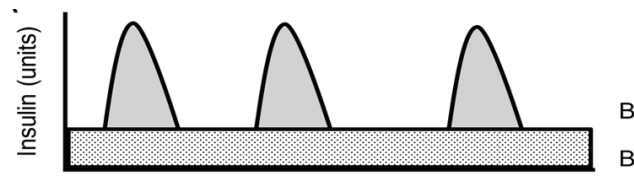

Bolus insulin

Basal insulin
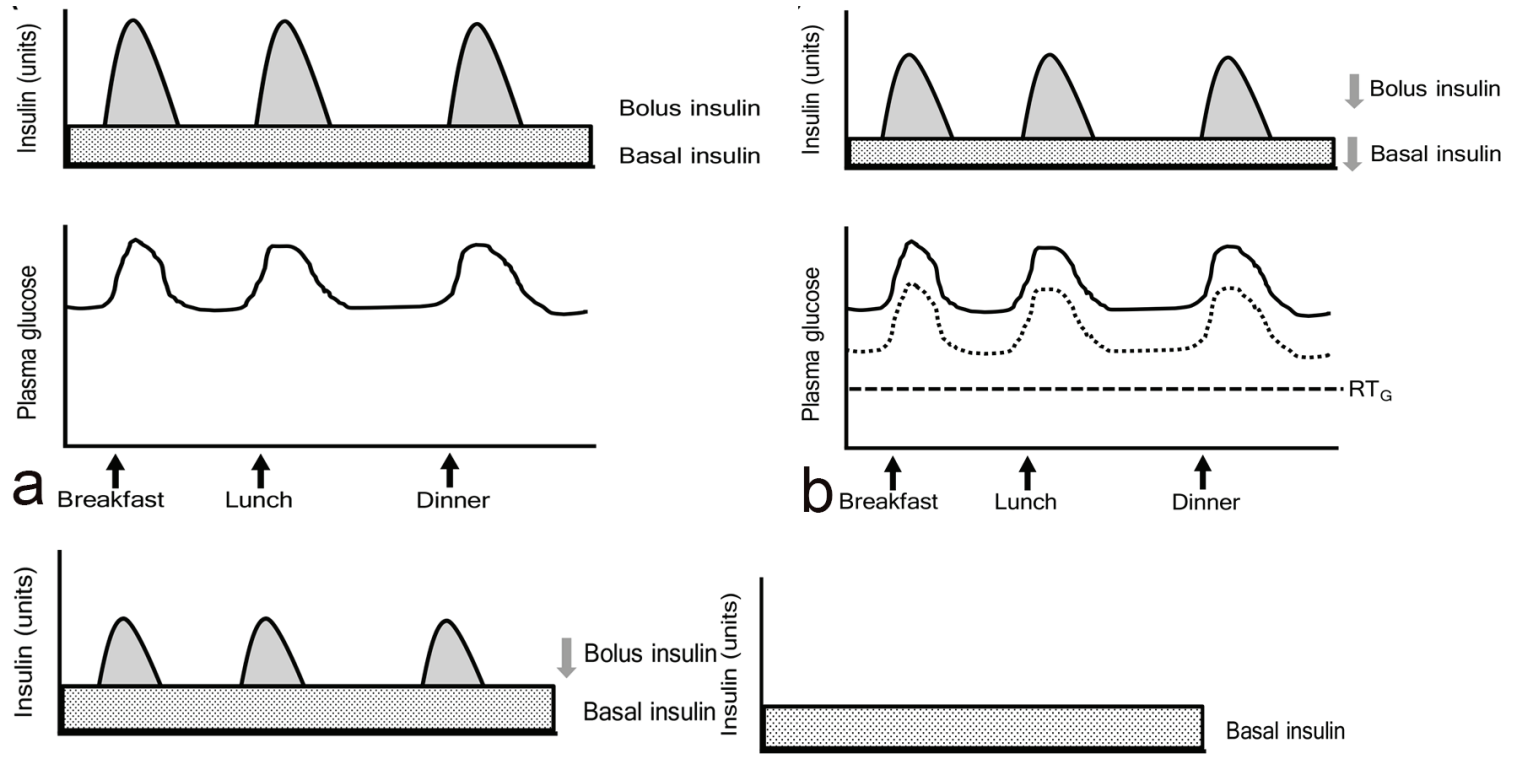

Basal insulin
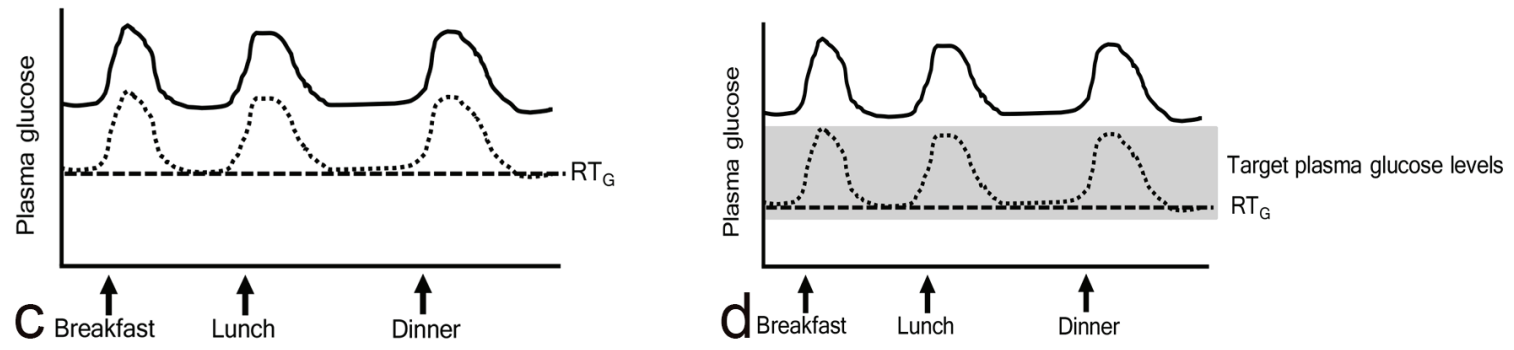

Figure 3. The association among bolus and basal insulin dose, plasma glucose and the renal threshold for glucose excretion $\left(\mathrm{RT}_{\mathrm{G}}\right)$ in patients treated with insulin and SGLT2 inhibitors. Solid and dotted lines indicate plasma glucose levels before and after the addition of SGLT2 inhibitors, respectively.

cerning this article.

\section{References}

1. Suzuki K, Mitsuma Y, Sato T, Anraku T, Hatta M. Comparison of combined tofogliflozin and glargine, tofogliflozin added to insulin, and insulin dose-increase therapy in uncontrolled type 2 diabetes. J Clin Med Res. 2016;8(11):805-814.

2. Henry RR, Strange P, Zhou R, Pettus J, Shi L, Zhuplatov SB, Mansfield T, et al. Effects of dapagliflozin on 24hour glycemic control in patients with type 2 diabetes: a randomized controlled trial. Diabetes Technol Ther. 2018;20(11):715-724.

3. Yamada T, Shojima N, Noma H, Yamauchi T, Kadowaki T. Sodium-glucose co-transporter-2 inhibitors as add-on therapy to insulin for type 1 diabetes mellitus: Systematic review and meta-analysis of randomized controlled trials. Diabetes Obes Metab. 2018;20(7):1755-1761.

4. Tang H, Cui W, Li D, Wang T, Zhang J, Zhai S, Song Y. Sodium-glucose co-transporter 2 inhibitors in addition to insulin therapy for management of type 2 diabetes mellitus: A meta-analysis of randomized controlled trials. Dia- betes Obes Metab. 2017;19(1):142-147.

5. Sha S, Devineni D, Ghosh A, Polidori D, Chien S, Wexler D, Shalayda K, et al. Canagliflozin, a novel inhibitor of sodium glucose co-transporter 2, dose dependently reduces calculated renal threshold for glucose excretion and increases urinary glucose excretion in healthy subjects. Diabetes Obes Metab. 2011;13(7):669-672.

6. Devineni D, Morrow L, Hompesch M, Skee D, Vandebosch A, Murphy J, Ways K, et al. Canagliflozin improves glycaemic control over 28 days in subjects with type 2 diabetes not optimally controlled on insulin. Diabetes Obes Metab. 2012;14(6):539-545.

7. Henry RR, Rosenstock J, Edelman S, Mudaliar S, Chalamandaris AG, Kasichayanula S, Bogle A, et al. Exploring the potential of the SGLT2 inhibitor dapagliflozin in type 1 diabetes: a randomized, double-blind, placebo-controlled pilot study. Diabetes Care. 2015;38(3):412-419.

8. Pieber TR, Famulla S, Eilbracht J, Cescutti J, Soleymanlou N, Johansen OE, Woerle HJ, et al. Empagliflozin as adjunct to insulin in patients with type 1 diabetes: a 4-week, randomized, placebo-controlled trial (EASE-1). Diabetes Obes Metab. 2015;17(10):928-935.

9. Matsumura M, Nakatani Y, Tanka S, Aoki C, Sagara M, Yanagi K, Suzuki K, et al. Efficacy of additional canagli- 
flozin administration to type 2 diabetes patients receiving insulin therapy: examination of diurnal glycemic patterns using Continuous Glucose Monitoring (CGM). Diabetes Ther. 2017;8(4):821-827.

10. Hakoshima M, Yanai H, Kakuta K, Adachi H. SodiumGlucose Cotransporter 2 Inhibitors Reduce Prandial Insulin Doses in Type 2 Diabetic Patients Treated With the Intensive Insulin Therapy. J Clin Med Res. 2018;10(6):493-498.

11. Rosenstock J, Jelaska A, Zeller C, Kim G, Broedl UC, Woerle HJ, investigators E-RBt. Impact of empagliflozin added on to basal insulin in type 2 diabetes inadequately controlled on basal insulin: a 78-week randomized, double-blind, placebo-controlled trial. Diabetes Obes Metab. 2015;17(10):936-948.

12. Terauchi Y, Tamura M, Senda M, Gunji R, Kaku K. Longterm safety and efficacy of tofogliflozin as add-on to insulin in patients with type 2 diabetes: Results from a 52week, multicentre, randomized, double-blind, open-label extension, Phase 4 study in Japan (J-STEP/INS). Diabetes Obes Metab. 2018;20(5):1176-1185. 